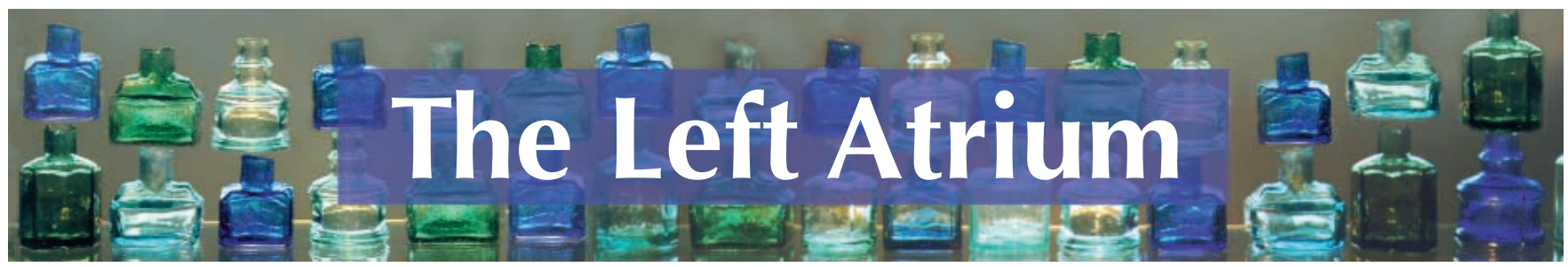

\section{More than getting out of the cold}

The health care of homeless persons: a manual of communicable diseases and common problems in shelters and on the streets James J. O'Connell, editor

Boston, Mass: Guthrie Nixon Smith; 2004 345 pp US\$15 ISBN 0-9711650-8-4

W ith tens of thousands of people in Canada estimated to be homeless on any given night, ${ }^{1}$ many Canadian health care workers frequently face the challenge of caring for homeless patients. But is health care for this population so distinct that we need a separate manual to guide us? An answer is given in the editor's introduction to this book:

Caring for homeless persons requires a deliberate blurring of the traditional boundaries between institutions and among health care disciplines and professions. While physicians, nurse practitioners, and physician assistants can diagnose and treat according to Harrison's Principles of Internal Medicine, a homeless person with a lower extremity ulcer and cellulitis needs access to antibiotics and a place to keep the leg elevated, an impossibility while living in shelters or on the streets. Thus the clinician's treatment plan is only as effective as the social worker who obtains the medications, the nurses who perform the twice daily dressing changes, and the shelter supervisor who permits the person to remain in the lobby during the day.

This manual was produced by the Boston Health Care for the Homeless Program (BHCHP), a project initiated in the 1980s "to provide or assure access to the highest quality health care for homeless individuals and families in Boston." The BHCHP currently consists of multiple services, including 3 hospital-based clinics, a multidisciplinary team providing care at over 70 outreach and community sites throughout Boston, oral health care at a dental clinic and dental suites, and a respite program.

Drawing on two decades of experience of the BHCHP and the expertise

of other health care workers throughout the United States, this manual provides practical insight into the challenges of caring for homeless people. It has a vast scope, ranging from the prevention and management of influenza infection to preparing for a bioterrorist attack. The book is divided into broad sections on communicable disease, exposure-related conditions, emerging challenges in street and shelter medicine, chronic disease management, immunization and food management. A final section consists of a series of fact sheets (provided in English and Spanish) on communicable diseases such as hepatitis $\mathrm{C}$ and infectious diarrhea, intended for distribution to shelter guests and their families in the event of an outbreak.

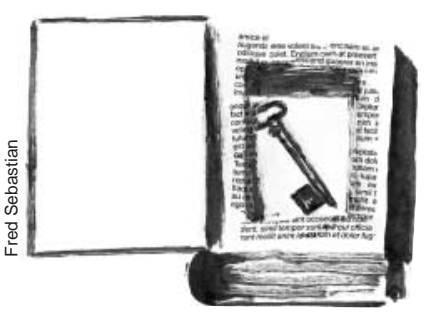
viding directly observed tuberculosis therapy on Boston's streets. The images leave the reader with a sense of the harshness of being homeless, and of the commitment and creativity of those who provide care to this population.

Certain aspects of the manual may be more applicable to health care in the US than to practice in Canada. Much of the epidemiologic data on specific conditions and on homelessness focuses on the US context. The fact sheets on communicable diseases are provided in English and Spanish, whereas a French version would be useful in Canada. Also, a guide to the costs of relevant medications is provided, which may differ from the costs, absolute and relative, of the same medications in Canada.

Nonetheless, this manual is a helpful aid for anyone involved in clinical work or other types of service provision for homeless people. Although some of the information it provides is specific to the US, it remains valuable as a resource for Canadian practitioners on specific clinical conditions as they affect people who are homeless, and on general principles about caring for this popula-

The clinical chapters provide concise discussions of epidemiology, diagnosis and the management of various conditions, adapted for the needs of people who are homeless. These chapters successfully integrate, as required, approaches that have traditionally been separated into public health and medical spheres.

Among the assets of the manual are excellent clinical images illustrating conditions that affect people who are homeless, such as frostbitten toes and cellulitis, and including up-close (and itch-inducing) photographs of Cimex lectularius (bed bugs) and Pediculus humanus (lice). Photographs also show BHCHP staff engaged in various tasks ranging from political advocacy to pro- tion. Of course, the stark truth about the manual is laid out by editor James J. O'Connell in his acknowledgements:

If safe and affordable housing and access to quality health care were available for all, most of the communicable diseases and medical conditions described in this manual would be rare and easily managed.

\section{Fiona Kouyoumdjian}

Medical Student

Dalhousie University

Halifax, NS

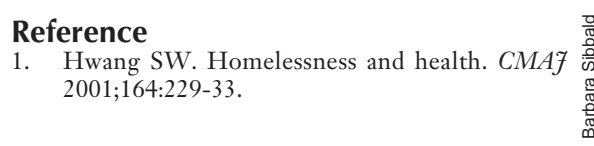

